\title{
Research on Network Resource (Bandwidth) Reservation
}

\author{
Wenpeng Cao*, Xinchang Zhang, Jiedong Bi \\ Shandong Computing Center, Qilu University of Technology, China \\ ${ }^{*}$ Corresponding author. Email: goodcwp@163.com
}

\begin{abstract}
This paper proposes two solutions about bandwidth reservation. On the one hand, the algorithm strategy of network resource bandwidth reservation is considered, and on the other hand, the scheduling method of bandwidth reservation for multi-user and multi-task is considered. In the process of bandwidth transmission, attention to a single request task can be placed on the user as a whole, so that the user's bandwidth scheduling can be optimized. In addition, the network bandwidth scheduling table on the transmission path is divided into a batch of bandwidth allocatable areas, which can be used to schedule task requests iteratively when processing bandwidth scheduling request tasks.
\end{abstract}

Keywords: Network, Bandwidth, Reservation.

\section{INTRODUCTION}

With the continuous development of computer network, work and life in a variety of network-related business needs are also growing on a large scale. Due to the limited network capacity, along with the improvement of users' business needs, the management ability and quality problems of network resources when facing individuals also increase, which makes it difficult to guarantee the requirements of quality of service (QoS).Therefore, in view of the current network problems, how to change the existing network architecture to adapt to the needs of contemporary development is particularly important.

Resource reservation means that network resources such as links and ports are reserved in advance for applications with QoS requirements. QoS requirements mean that there are certain requirements on link bandwidth, path delay and packet loss rate. Moreover, during the operation of these services, these resources are only allowed to be used by users who put forward the reservation, and the QoS of the services cannot be satisfied because of network congestion, nor can they be affected by other services because of resource competition. Traditional networks provide network services as best they can. Although there is a resource reservation protocol [1], the implementation of this protocol is relatively complicated, so it is not widely used. The closure and uncontrollability of traditional networks make it impossible for us to know the utilization of network resources, let alone control the allocation and use of resources.

Resource reservation mechanism is to reserve network resources in OBS network by means of sending control group when dealing with data burst. According to the process of resource reservation, it can be divided into "one-step resource reservation" and "two-step resource reservation". One-step resource reservation means that after the control packet is sent, the corresponding data burst does not need to wait for the confirmation message of whether the resource reservation is successful, but only needs to wait for a bias time before the message can be delivered through the data path. Two-step resource reservation requires a data burst to be sent after a confirmation message that the resource reservation is successful is received.

There are many ways to implement the resource reservation mechanism, which can be divided into four categories according to the time and way of resource reservation and resource release:

(1) Explicit reservation, explicit release

(2) Explicit reservation, estimated release

(3) Estimated reservation, explicit release

(4) Estimated reservation, estimated release

Bandwidth reservation is the process of allocating bandwidth to users and network services. In order to improve the utilization of network resources, to achieve 
the maximum use of network resources. When facing different business needs, different priority policies are set according to the business importance and the response time of network delay. In the case of network problems, the priority method is used to release those blocked networks and optimize network allocation.

\section{RELATED WORK}

\subsection{Research Method of Bandwidth Reservation}

In the process of resource reservation, the problem of path selection should be taken into full consideration. In the current routing algorithms, such as the shortest routing algorithm [2][3], genetic algorithm [4], etc. In the following discussion, this paper mainly considers ant colony algorithm [5].In the traditional ant colony algorithm, the main consideration is to solve the optimization problem, does not involve the QoS problem. Therefore, it is necessary to take into account the improvement of the traditional ant colony algorithm to meet the requirements of QoS and the influence factors of other applications to achieve the new ant colony algorithm that meets the research content when designing the combination algorithm.

Ant colony algorithm in MarcoDorigo in 1992 [6] the thesis raises, found by walking paths when ants looking for food behavior, when the ant search path forward, based on the information of the other ants stay ahead of the have to choose their own path to walk, the ant to choose a path and the path pheromone intensity is proportional to the relationship. The behavior of ant colony to find food according to the path can be considered as information learning. The characteristic of this learning mode is that the more information on a certain path, the more likely it is to be passed by other information in the subsequent process.

Classical ant colony algorithm [7] for the purpose of this article has certain limitations, to solve the problem of algorithm does not involve the QoS constraints, such as time delay and bandwidth, the path of the selected cannot satisfy the QoS requirements of the business, and the ants in a pathfinding process will iterate through all the network nodes, it is not necessary for this article, The path search can be ended as long as the path traveled by the ant contains the source node and the destination node, and the path search can be ended in advance when the delay of the path is greater than the maximum delay required by the application. In addition, when the network is congested, in order to ensure the bandwidth demand of the service and balance the load of the network, we can seek multiple paths for one stream and distribute the data flow to multiple paths.

Ant Colony Optimization of Bandwidth Reservation (BRACO), the bandwidth of the network application request delete dynamic network topology does not meet the requirements of the bandwidth of the link, thus forming the new network topology relationship and generate the corresponding with the adjacency list. Based on the ant colony algorithm model, a fixed number of ants $\mathrm{M}$ only search for paths alone, and will stop only when the end condition of finding paths is met. All the collected path $\mathrm{n}(\mathrm{n}<=\mathrm{m})$ the combination of the objective function to select the optimal path set, finally, pheromone is added to each link of the optimal path set, and pheromone is reduced in other paths. Then, the above path finding process is repeated for iterative calculation according to all paths at this time, until the required maximum number of iterations is finally met.

For the bandwidth acceptance problem, bandwidth reservation is an important aspect of network acceptance strategy. The traditional way of bandwidth acceptance only focuses on the acceptance of the whole network resources, but it is difficult for the acceptance strategy of network traffic to achieve fair distribution when facing different bandwidth demands in the actual network. In Diffserv's paper [8], a hierarchical bandwidth reservation acceptance strategy is proposed to solve the fairness problem. For the study of this problem, Measuredsum [9] also introduced the classical acceptance algorithm and analyzed the idea and the fairness of acceptance reservation in detail.

Tan.X.H et al. [10] realized an effective bandwidth calculation method by studying IntServ resource reservation. The calculation method of resource reservation is obtained based on network performance calculation and derivation calculation, and the accuracy of the experiment is verified from two aspects of bandwidth delay and bandwidth constraint combined with practical network application.

\subsection{Bandwidth Reservation Scheduling Policy}

The mode of bandwidth scheduling can be divided into the following two kinds: (1) Instant scheduling: dispatching immediately after receiving a request; (2) Cycle scheduling: scheduling the multiple requests accumulated in a specific time interval as a whole. The ISP wants to successfully schedule all incoming requests to improve system throughput and resource utilization. The study of just-in-time scheduling focuses on the performance of a single BRR such as the earliest completion time and the shortest duration, while cycle scheduling focuses on the overall performance of the batch of requests over a time interval.

Balman et al. considered the earliest completion time and the shortest duration in immediate scheduling [11]. After receiving the user's request, they traverse all time Windows within the expected time interval and select the option with the earliest end time and the maximum bandwidth. The complexity of these problems is analyzed 
in detail, and the corresponding algorithms are proposed. Tongshu and $\mathrm{Wu}$ formulated a real-time bandwidth scheduling problem: minimize the energy consumption under the constraints of data transmission term under the premise of scheduling completion. The authors adopted a practical power model evaluation, and proposed an algorithm for polynomial time optimal solution for the model [12].Wang et al. developed a problem of maximizing the total bandwidth: maximizing the total bandwidth of $\mathrm{k}$ paths with disjoint edges, where $\mathrm{k}>1$.In addition, the network resources are specially defined so that each path can improve the bandwidth utilization rate and thus increase the total bandwidth [13].Liu Jing et al. proposed a network multi-path transmission mode based on independent spanning tree, and analyzed the network transmission performance in terms of transmission time and transmission speed [14].As a greedy algorithm, Dijkstra's maximum bandwidth algorithm is used to schedule BRR in turn. Dijkstra's maximum bandwidth algorithm is the optimal solution when dispatching a single BRR in time. Periodic scheduling algorithms generally arrange BRR in some order other than the receiving order of BRR. For example, Literature [15] uses the descending order of $\mathrm{D}$. This is not suitable for real-time scheduling where BRR information is unknown. FixBW algorithm draws on the idea of literature [13], which regards multiple paths as multiple requests. The original text selects the path with the lowest resource score by using multiple paths calculated in a batch. T.Wang et al. [16] choose the path with bandwidth equal to $\mathrm{D}\left(t^{e}-t^{s}\right)$, that is, they tend to choose the option that makes full use of the current link bandwidth.Try to avoid the link with small bandwidth and try to leave the link with large bandwidth, which is conducive to the subsequent user request scheduling.

In the bandwidth scheduling problem of high performance networks, it is mainly divided into two aspects: immediate bandwidth and cycle bandwidth. Cycle bandwidth can be divided into similar data transmission and heterogeneous data transmission problems. For the study of real-time scheduling, in the literature [17], the author studied the high-throughput scientific workflow scheduling algorithm under certain constraints in high-performance networks. Wu and Lin combined the bandwidth and path restrictions. Four kinds of bandwidth scheduling problems are proposed: (1) fixed path bandwidth, (2) fixed path bandwidth, (3) fixed path bandwidth, (4) fixed path bandwidth. For each task request, the four questions above have the same goal, which is to minimize the transfer time. In Literature [18], N.Rao et al. demonstrated four basic scheduling problems for different target bandwidth and time slot restrictions, and proposed the corresponding more efficient bandwidth scheduling algorithm. The first three problems can be solved by Dijkstra's expansion algorithm, and the last problem needs to be solved by Bell-Ford algorithm. Literature [19] also studied similar problems. Based on the research of $\mathrm{Wu}$ and N.Rao, detailed solutions to these four problems were given, and the advantages of algorithm performance were evaluated. In Literature [20], Grimmell et al. proposed a dynamic and fast path finding method, which can make the path from source node to destination node. Data transmission has a minimum delay, for a batch of data transmission requests, is conducive to the improvement of the success rate of bandwidth scheduling. In Literature [21], the author analyzes the problem of bandwidth scheduling under several different classifications in view of the changes of path and bandwidth in high-performance networks and whether the time-delay of switching path is negligible, and gives the optimal algorithm and heuristic algorithm. These algorithms are designed for single-user task requests. It is difficult to apply to the high performance network bandwidth request of multi-user and multi-task.

The above mentioned research mainly focus on the HPN real-time scheduling problem, in order to further improve the utilization rate of network resources, scheduling, the researchers focused on the cycle of the so-called cycle scheduling, refers to when a task, the scheduler will not immediately for scheduling, task be accumulated in the specified size cycle storage, after a period, the scheduler then schedules these tasks. Lin and Wu pointed out in the article [22] that in highperformance private network, it is very important to efficiently find the appropriate path and bandwidth allocation scheduling for the full utilization of network resources and the needs of users. The author proposed the problems of MDTA and MFBR, both of which are to schedule a batch of requests within a specified period. MDTA problem refers to that users want their data to be transmitted as soon as possible, and there is no need to specify the bandwidth size and transmission time gap of the task, so as to minimize the total transmission time of users. And MFBR problem refers to each request has its minimum reserved bandwidth, start time and end time, for every request, to find a path from source node to destination node, if available, is to update the edge of the path of the state table, the problems of this approach is that cannot guarantee that all the reserved bandwidth request can be satisfied, this paper solves the problem that the bandwidth reservation of users can be maximized in a given topology and user request. In the paper [23], Zuo studied how to make the network's limited network bandwidth resources meet the task requests as much as possible when a batch of task requests occur concurrently in the high-performance network, and at the same time minimize AECT(average earliest completion time) and ASD(average shortest completion time).In this paper, the authors prove that ASD and AECT belong to NP-hard problems, and give corresponding solving algorithms. This paper solves a batch of task requests. When the task requests are multi-user, this method has a low success rate of scheduling and cannot solve this situation well. 


\section{CONCLUSION}

Due to the continuous update of information technology, the rapid development of big data and cloud platform, the traditional network is difficult to cope with the challenge of such unprecedented large-scale data transmission tasks, which will surely promote the emergence of high-performance network, especially in the field of public network and private network infrastructure. This trend is already evident in public networks. Typical examples include Internet2 and ESnet, the U.S. Department of Energy's main network, which use OSCARs technology to provide IP-based multiprotocol label switching (MPLS) tunneling and virtual private network (VLAN) services. Therefore, highperformance networks have great development potential, and a large number of high-quality related studies are needed to make expensive computing resources and network resources play their real role.

In the process of solving the problem of network bandwidth resources reserve, put forward the corresponding bandwidth algorithm and scheduling strategy, has better performance on improving performance, but in different environment for different bandwidth request task, due to the choice of bandwidth algorithm and the frame of the bandwidth of the network itself are connected, so on the choice should be according to the algorithm and bandwidth request characteristics make reasonable choice.

At the same time, this paper still has a lot of work to continue to do further research, can be mainly expanded in the following three aspects, first, there are many algorithms in bandwidth reservation can be optimized and innovated; Secondly, in the aspect of bandwidth scheduling, we can combine bandwidth scheduling with path selection for further research. Finally, the method of bandwidth reservation itself has a lot of optimization improvements, which can further improve the execution efficiency of the method and strategy.

\section{REFERENCES}

[1] Zhou Qi, Deng Zhicheng, Zhu Xianghua. Internet Resource Reservation Protocol-RSVP [J]. Data Communication, 1999, 3.

[2] Yang Yun, JOE, Li Qianmu, et al. Multi-objective QoS routing algorithm based on Dijkstra policy [J]. Miniature Microcomputer System, 2004, 25(9):1660-1664.

[3] Wroclawski J. Specification of the controlled-load network element service[J]. 1997.

[4] Sun B, Li L, Chen H. Shortest path routing optimization algorithms based on genetic algorithms[J]. Computer Engineering, 2005, 31(6): 142-144.
[5] Sun Y, Ma H D, Liu L. An ant-colony optimization based service aware routing algorithm for multimedia sensor networks[J]. Dianzi Xuebao (Acta Electronica Sinica), 2007, 35(4): 705-711.

[6] Dorigo M. Using transputers to increase speed and flexibility of genetics-based machine learning systems[J]. Microprocessing and Microprogramming, 1992, 34(1-5): 147-152.

[7] Huang Guorui, Cao Xianbin, Wang Xufa.Ant Colony Algorithm Based on Pheromone Diffusion [D].,2004.

[8] Peng J. A Classified and Bandwidth-Reserved Admission Control Algorithm and Analysis of its Fairness[J]. Computer Engineering and Applications, 2004, 4.

[9] Lai Y C, Tsai S F. Unfairness of measurement-based admission controls in a heterogeneous environment[J]. International Journal of Communication Systems, 2001, 14(9): 871-883.

[10] Tan X H, Gao X, Jin W D. On method of network resource reservation based on network calculus[J]. Application Research of Computers, 2007: 11.

[11] Balman M, Chaniotakisy E, Shoshani A, et al. A flexible reservation algorithm for advance network provisioning[C]//SC'10: Proceedings of the 2010 ACM/IEEE International Conference for High Performance Computing, Networking, Storage and Analysis. IEEE, 2010: 1-11.

[12] Shu T, Wu C Q, Yun D. Advance bandwidth reservation for energy efficiency in highperformance networks[C]//38th Annual IEEE Conference on Local Computer Networks. IEEE, 2013: 541-548.

[13] Wang T, Wu C Q, Wang Y, et al. Multi-Path Routing for Maximum Bandwidth with K EdgeDisjoint Paths[C]//2018 14th International Wireless Communications \& Mobile Computing Conference (IWCMC). IEEE, 2018: 1178-1183.

[14] Liujing, Fan Jianxi. Research on Network Multipath Transmission Method Based on Independent Spanning Tree [J]. Software, 2016 (April 2016): 2528.

[15] Zuo L, Zhu M M, Wu C Q. Bandwidth reservation strategies for scheduling maximization in dedicated networks[J]. IEEE Transactions on Network and Service Management, 2018, 15(2): 544-554.

[16] Wang Tao, Wang Yongqiang, Alva Wang. The bandwidth reservation strategy of maximizing periodic scheduling in real-time scheduling [J]. Software, 2019, 040(012):118123. 
[17] Lin Y, Wu Q. On design of bandwidth scheduling algorithms for multiple data transfers in dedicated networks[C]//Proceedings of the 4th ACM/IEEE Symposium on Architectures for Networking and Communications Systems. 2008: 151-160.

[18] Rao N S V, Wu Q, Ding S, et al. Control plane for advance bandwidth scheduling in ultra high-speed networks[C]//Proceedings IEEE INFOCOM 2006. 25TH IEEE International Conference on Computer Communications. IEEE, 2006: 1-5.

[19] Brown P, Zhu M, Wu Q, et al. Exploring the optimal strategy for large-scale data movement in highperformance networks[C]//2012 IEEE 31st International Performance Computing and Communications Conference (IPCCC). IEEE, 2012: 181-182.

[20] Dharam P, Wu Q. Advance bandwidth reservation with delay guarantee in high-performance networks[C]//2012 21st International Conference on Computer Communications and Networks (ICCCN). IEEE, 2012: 1-7.

[21] Banerjee A, Feng W, Mukherjee B, et al. RAPID: An end-system aware protocol for intelligent data transfer over lambda grids[C]//Proceedings 20th IEEE International Parallel \& Distributed Processing Symposium. IEEE, 2006: 10 pp.

[22] Sahni S, Rao N, Ranka S, et al. Bandwidth scheduling and path computation algorithms for connection-oriented networks[C]//Sixth international conference on networking (ICN'07). IEEE, 2007: 47-47.

[23] Zuo L, Zhu M M. Concurrent bandwidth reservation strategies for big data transfers in high-performance networks[J]. IEEE Transactions on Network and Service Management, 2015, 12(2): 232-247. 